Acta Crystallographica Section F

Structural Biology

and Crystallization

Communications

\section{addenda and errata}

ISSN 1744-3091

\title{
Production, crystallization and X-ray diffraction analysis of two nanobodies against the Duffy binding-like (DBL) domain DBL6e-FCR3 of the Plasmodium falciparum VAR2CSA protein. Corrigendum
}

\author{
Anneleen Vuchelen, ${ }^{a, b}$ Els Pardon, ${ }^{a, b}$ Jan Steyaert, ${ }^{a, b}$ \\ Benoît Gamain, ${ }^{\mathrm{c}, \mathrm{d}, \mathrm{e}, \mathrm{f}}$ Remy Loris, ${ }^{\mathrm{a}, \mathrm{b}_{*}}$ Nico A. J. van \\ Nuland $^{a, b}$ and Stéphanie Ramboarina ${ }^{a, b_{*}}$

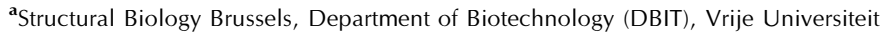 \\ Brussel, Pleinlaan 2, B-1050 Brussels, Belgium, ${ }^{\mathbf{b}}$ Molecular Recognition Unit \\ (MoRe), Department of Structural Biology, VIB, Pleinlaan 2, B-1050 Brussels, \\ Belgium, 'Inserm, UMRS665, F-75015 Paris, France, 'Université Paris Diderot, \\ F-75013 Paris, France, ${ }^{\mathbf{e}}$ Institut National de la Transfusion Sanguine, F-75015 Paris, \\ France, and 'Laboratoire d'Excellence Gr-Ex, France \\ Correspondence e-mail: reloris@vub.ac.be, stephanie.ramboarina@cea.fr
}

A correction is made to Fig. 1 and the text of the article by Vuchelen et al. (2013, Acta Cryst. F69, 270-274).

In the article by Vuchelen et al. (2013) there is a mistake in Fig. 1. The second line of $\mathrm{Nb} 19$ is a copy of the second line of $\mathrm{Nb} 07$, instead of the correct $\mathrm{Nb} 19$ line. This results in a difference of four amino acids. In $\S 3$, it is mentioned that there is one amino-acid substitution between the two nanobodies but this should be five (to include the four from the second line). Also the sequence identity should be $94 \%$ instead of $98 \%$. A correct version of Fig. 1 is published here.

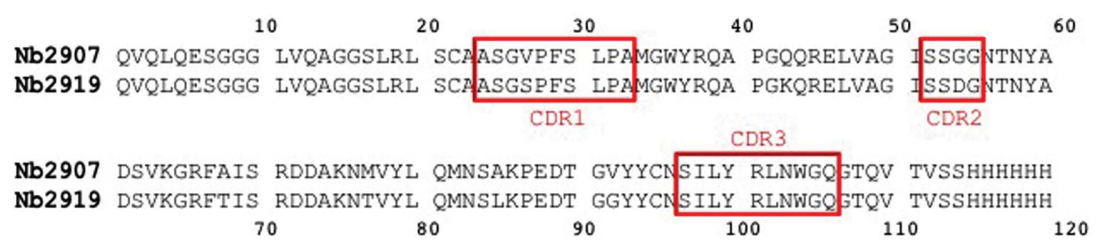

Figure 1

Corrected sequence alignment of $\mathrm{Nb} 2907$ and Nb2919. The complementary determining regions $(\mathrm{CDR})$ are shown in red boxes.

\section{References}

Vuchelen, A., Pardon, E., Steyaert, J., Gamain, B., Loris, R., van Nuland, N. A.

J. \& Ramboarina, S. (2013). Acta Cryst. F69, 270-274. 